\title{
Intravenous Immunoglobulins as Adjunct Treatment to Phototherapy in Isoimmune Hemolytic Disease of the Newborn: A Retrospective Case-Control Study
}

\author{
Manar Al-lawama a, b, Eman Badran a , Ala' Elrimawia, \\ Amal Bani Mustafa ${ }^{a}$, Haitham Alkhatiba
}

\begin{abstract}
Background: Isoimmune hemolytic disease is a major cause of neonatal severe indirect hyperbilirubinemia that requires phototherapy or exchange transfusion which is an invasive procedure to avoid brain injury. Administration of intravenous immunoglobulin (IVIG) is used as an adjunct treatment to phototherapy in order to decrease the rate of exchange transfusion.
\end{abstract}

Methods: This retrospective case-control study aimed to describe the safety and efficacy of IVIG therapy in newborns with isoimmune hemolytic disease and to compare their clinical outcomes to those of a control group who were treated only with phototherapy. Criteria for IVIG treatment were variable; when phototherapy threshold was reached or when exchange transfusion level was approached, using either indication is based on the attending discretion.

Results: Ninety-four infants were included in the IVIG group, compared to 108 infants in the control group. Most of the included infants were term infants and most common cause was $\mathrm{ABO}$ incompatibility. There were no side effects documented in all the included infants. The IVIG group had more severe hemolysis with average highest bilirubin of $14.6 \pm 3.7 \mathrm{mg} / \mathrm{dL}$ in the IVIG group versus $12.6 \pm 3$ in the control group $(\mathrm{P}=0.0001)$. Complication of hemolysis was seen more in the IVIG group with higher rate of rebound hyperbilirubinemia, blood transfusion and exchange transfusion.

Conclusions: IVIG use as an adjunct treatment to phototherapy in isoimmune hemolytic disease of the newborns is safe. The favorable results of the phototherapy only group were supportive of using selective criteria for administration of IVIG in neonates with isoimmune hemolytic disease.

Keywords: Neonate; Intravenous immunoglobulin; Isoimmune hemolytic disease; ABO incompatibility; Rh isoimmunization

Manuscript submitted September 14, 2019, accepted September 30, 2019

aJordan University Hospital, The University of Jordan, Amman, Jordan ${ }^{b}$ Corresponding Author: Manar Al-lawama, Jordan University Hospital, The University of Jordan, Queen Rania Street, Amman 11943, Jordan.

Email: manar-76@hotmail.com

\section{Introduction}

Isoimmune hemolytic disease of the newborn is caused by incompatibility between maternal and fetal major or minor blood groups, or it can be caused by Rhesus incompatibility [1]. It is the major cause of severe indirect hyperbilirubinemia in the neonatal period [2].

The diagnosis is usually made by the presence of blood group incompatibility between mother and fetus along with a positive direct antibody test (DAT) [3]. The rate of bilirubin rise and the drop in hemoglobin level is used to document the presence of hemolysis and to guide treatment modalities [4].

Phototherapy is the main treatment modality [5]. It alters the solubility of the indirect bilirubin by isomerization of the bilirubin compound to make it water soluble [6]. The administration of intravenous immunoglobulin (IVIG) is an adjunctive treatment by blocking of the reticuloendothelial Fc receptor sites and thereby preventing the extravascular destruction of neonatal red blood cells (RBCs) sensitized by maternal antibodies $[7,8]$. It was shown to decrease the need for blood exchange transfusion $[9,10]$.

The use of IVIG in isoimmune hemolytic disease of the newborn is guided adopting international guidelines such as the American Academy of Pediatrics' Hyperbilirubinemia Guidelines [11], or it can be guided by nationally or locally drafted guidelines.

There is a wide range in the incidence and variety of side effects caused by the administration of IVIG. Its usage has been linked with several potential adverse reactions including fever, skin rash, cyanosis, hypotension, hypothermia, irritability and vomiting [12]. More recently, an association with necrotizing enterocolitis has also been described [13]. However, IVIG has been deemed safe, and is generally well tolerated with a limited side effect profile [7-10, 14, 15].

This is a retrospective case-control study that investigated the clinical outcomes of neonates with isoimmune hemolytic disease who have received phototherapy and IVIG compared to matched controls that were treated with phototherapy alone.

\section{Materials and Methods}

This retrospective case-control study was conducted to investi- 
Table 1. Demographic and Clinical Characteristics of Neonates With Isoimmune Hemolytic Disease According to Their Treatment Group

\begin{tabular}{llll} 
Characteristics & IVIG group $(\mathbf{n = 9 4 )}$ & Phototherapy group $(\mathbf{n}=\mathbf{1 0 8})$ & P value \\
\hline Gestational age & $37.9 \pm 1.6$ & $38.2 \pm 1.6$ & 0.18 \\
Birth weight & $3,022 \pm 462$ & $3,057 \pm 511$ & 0.16 \\
Male gender & $48(51 \%)$ & $50(46 \%)$ & 0.57 \\
Cesarean section & $47(50)$ & $36(33 \%)$ & $0.02 *$ \\
Incompatibility & & & $11(10 \%)$ \\
$\quad$ Rhesus & $21(22 \%)$ & $92(85 \%)$ & $0.02 *$ \\
$\quad$ Major blood group (ABO) & $69(73 \%)$ & $2(2 \%)$ & 0.04 \\
$\quad$ Minor blood group & $4(4 \%)$ & 0.31 \\
\hline
\end{tabular}

IVIG: intravenous immunoglobulin. *P value $\leq 0.5$.

gate the clinical outcomes of neonates with isoimmune hemolytic disease who received IVIG therapy, admitted to Jordan University Hospital, a tertiary care center with a specialized neonatal intensive care unit with a 30 bed capacity.

This study was approved by the deanship of scientific research at the University of Jordan. This study received ethical approval from the IRB and ethical committee at Jordan University Hospital. All procedures performed in studies involving human participants were in accordance with the ethical standards of the institutional and/or national research committee and with the 1964 Helsinki Declaration and its later amendments or comparable ethical standards. For this type of study formal consent is not required.

All neonates with isoimmune hemolytic disease above 32 weeks of gestation who have received IVIG therapy and were admitted to the neonatal unit during the period of $2012-2015$ were included. They were matched to a group of infants who were admitted during the same period with isoimmune hemolytic disease and who were treated with phototherapy only. Neonates who have received intrauterine exchange transfusion were excluded.

Screening of newborns for isoimmunization was done by DAT from cord blood sampling. Newborns qualified for this screening if they were born to Rh negative mothers, mothers with blood group type $\mathrm{O}$, mothers with positive indirect antibody test (IAT) or mothers with unknown blood group.

Any newborn with positive DAT result will be followed by sequential monitoring of his/her serum bilirubin and hemoglobin levels every $4-6 \mathrm{~h}$ according to the degree of hemolysis.

Phototherapy will be initiated when the newborn's serum bilirubin level reaches phototherapy threshold according to the American Academy of Pediatrics phototherapy graphs [11]. After initiation of phototherapy, bilirubin will be monitored initially every $4 \mathrm{~h}$ until rate of rise is determined. If level of rise is slow, frequency of bilirubin monitoring will be decreased to every $12 \mathrm{~h}$. Phototherapy will be discontinued when bilirubin level is $2-3 \mathrm{mg} / \mathrm{dL}$ below phototherapy level. The infant will be monitored for rebound hyperbilirubinemia in hospital for $24-48 \mathrm{~h}$ after discontinuation of phototherapy.

In isoimmune hemolytic disease, IVIG will be administered in our unit according to two protocols according to the attending physician: either when the neonate's bilirubin value is only $2 \mathrm{mg} / \mathrm{dL}$ below the level of exchange, or once the bilirubin level reaches phototherapy range, indicating that the hemolysis is significant. Those neonates represent the IVIG group. The control group consisted of neonates with isoimmune hemolytic disease who were managed by phototherapy alone, as their bilirubin did not approach exchange level.

IVIG was administered in a $1.0 \mathrm{~g} / \mathrm{kg}$ dose over a 2 -h period. During administration the neonate will be on continuous cardiorespiratory monitor.

Rebound hyperbilirubinemia was defined as the rise of bilirubin level to phototherapy threshold after discontinuation of phototherapy.

Readmission was defined as the need for another admission of the newborn infant to the neonatal unit or the pediatric floor due to complications related to hyperbilirubinemia and/ or hemolysis after completion of the $24-48 \mathrm{~h}$ observation period following discontinuation of phototherapy during the first admission.

\section{Statistical analysis}

Data are presented as numbers and frequencies. A Fisher's exact test was used to compare groups. P values $\leq 0.05$ were considered statistically significant.

\section{Results}

A total of 202 neonatal infants with isoimmune hemolytic disease were identified. Ninety-four infants were in the IVIG group, and 108 infants were in the phototherapy only group. Most of the included infants were term infants and most common isoimmune cause was ABO incompatibility (Table 1). There were no side effects documented in all the included infants. The IVIG group had more severe hemolysis than the phototherapy only group. Average highest bilirubin was $14.6 \pm$ 3.7 in the IVIG group versus $12.6 \pm 3$ in the phototherapy only group $(\mathrm{P}=0.0001)$, and average lowest hemoglobin $(\mathrm{Hb})$ was $11.8 \pm 2.5$ in the IVIG group versus $13.1 \pm 2.5$ in the phototherapy only group $(\mathrm{P}=0.0003)$. Complications of hemolysis were 
Table 2. Clinical Outcomes of Neonates With Isoimmune Hemolytic Disease According to Their Treatment Group

\begin{tabular}{|c|c|c|c|}
\hline Outcome & IVIG group $(n=94)$ & Phototherapy group $(\mathrm{n}=108)$ & P value \\
\hline Duration of phototherapy (average \pm SD), days & $3.5 \pm 2.5$ & $2.4 \pm 1.8$ & 1.1 \\
\hline Length of hospital stay (average \pm SD), days & $6.7 \pm 4.1$ & $6.9 \pm 6.9$ & 0.8 \\
\hline Need for exchange transfusion & $4(4.2 \%)$ & 0 & $0.04 *$ \\
\hline Need for blood transfusion & $17(18 \%)$ & $4(3.7 \%)$ & $0.001^{*}$ \\
\hline Readmission for phototherapy & $2(2.1 \%)$ & $3(2.7 \%)$ & 0.7 \\
\hline Hemoglobin (average \pm SD) & $11.8 \pm 2.5$ & $13.1 \pm 2.5$ & $0.0003 *$ \\
\hline Lowest hemoglobin value & 5.4 & 6.7 & \\
\hline TSB mg/dL (average \pm SD) & $14.6 \pm 3.7$ & $12.6 \pm 3$ & $0.0001 *$ \\
\hline
\end{tabular}

IVIG: intravenous immunoglobulin; TSB: total serum bilirubin; SD: standard deviation. * $\mathrm{P}$ value $\leq 0.05$.

seen more in the IVIG group with rates of rebound hyperbilirubinemia, blood transfusion and exchange transfusion were $50 \%$ versus $28.7 \%, 18 \%$ versus $3.7 \%$, and $4.2 \%$ versus $0 \%$ respectively. Rate of suspected sepsis was significantly lower in the IVIG group while rate of culture positive sepsis was the same in the two groups (Table 2).

\section{Discussion}

This study presents a retrospective analysis of health outcomes of newborns admitted with isoimmune hemolytic disease. They were classified into two groups, IVIG group representing those with isoimmune hemolytic disease who were treated with phototherapy and IVIG, and the phototherapy only group representing newborns with isoimmune hemolytic disease who were treated with phototherapy only.

There were no significant side effects reported in all the newborns included in the study. None of the infants developed necrotizing enterocolitis which was previously reported as complication of IVIG therapy in the neonatal period [13].

The rate of suspected sepsis episodes was significantly higher in the phototherapy only group; however this difference disappeared when reviewing the results of blood cultures. Many studies have looked into the role of IVIG in neonatal sepsis [16], which showed marginal reduction in the occurrence of sepsis in neonates who received IVIG. However, investigating this part was not the objective of this study; therefore we did not look into infection risk factors that might explain the difference found.

Since we have two protocols for the administration of IVIG in isoimmune hemolytic disease, the IVIG group was a heterogeneous group of newborns because it consisted of neonates with severe hemolysis who were approaching or at exchange level when IVIG was administered, and those who received IVIG as soon as they reached phototherapy threshold which was considered as an indicator of their significant hemolysis.

The presence of the newborns with more severe hemolysis in the IVIG group was reflected on the incidence of complications and therapy modalities related to the hemolytic process. Lowest hemoglobin value, highest bilirubin value, rate of blood transfusion and blood exchange transfusion, and rate of rebound hyperbilirubinemia were all significantly higher in the IVIG group.

IVIG use was associated with the development of earlier and more severe anemia, which was shown clearly in our results as higher rate of blood transfusion in the IVIG group during first hospitalization (Table 2). IVIG increases the production of proinflammatory cytokines which in turn inhibits erythropoietin production [17]. However, the higher rate of inhospital anemia and blood transfusion in the IVIG group might be attributed to more severe hemolysis in newborns included. Late anemia in isoimmune hemolytic disease is hyporegenerative anemia. Several mechanisms have been proposed, including intramedullary destruction of $\mathrm{RBC}$ precursors by $\mathrm{RBC}$ antibodies, and erythropoietin production that is inappropriately low for the degree of anemia [18]. After discharge from the hospital, both groups had the same rate of late anemia and blood transfusion.

This study supports safe use of IVIG in isoimmune hemolytic disease of the newborns. The favorable results of the phototherapy only group were supportive of using selective criteria for administration of IVIG in neonates with isoimmune hemolytic disease even if they reached phototherapy threshold. Bilirubin rises despite intensive phototherapy [11] and significant hemoglobin drop can be used as indication to administer IVIG before the newborn approaches exchange level.

This study is a retrospective case-control study. Larger prospective studies with strict inclusion and follow-up criteria 
are needed to better understand the risks and benefits of IVIG administration as adjunctive management to phototherapy of isoimmune hemolytic disease of the newborns.

\section{Acknowledgments}

The authors are grateful to every member of the medical staff at Jordan University Hospital NICU who participated in the care of those infants.

\section{Financial Disclosure}

This study did not receive funding.

\section{Conflict of Interest}

The authors have no conflict of interest to declare.

\section{Informed Consent}

Informed consent is not requested as this study is a retrospective chart review. The authors are the treating physicians. The study did not involve identification of patients.

\section{Author Contributions}

MA contributed to the idea, design and writing; EB contributed to acquisition of data and approved the final draft; AE, $\mathrm{ABM}$ and HA gathered data, and were involved in analysis and writing.

\section{References}

1. Ullah S, Rahman K, Hedayati M. Hyperbilirubinemia in neonates: types, causes, clinical examinations, preventive measures and treatments: a narrative review article. Iran J Public Health. 2016;45(5):558-568.

2. Lopriore E, Rath ME, Liley H, Smits-Wintjens VE. Improving the management and outcome in haemolytic disease of the foetus and newborn. Blood Transfus. 2013;11(4):484-486.

3. Valsami S, Politou M, Boutsikou T, Briana D, Papatesta M, Malamitsi-Puchner A. Importance of Direct Antiglobulin Test (DAT) in cord blood: causes of DAT $(+)$ in a cohort study. Pediatr Neonatol. 2015;56(4):256-260.

4. Murray NA, Roberts IA. Haemolytic disease of the new- born. Arch Dis Child Fetal Neonatal Ed. 2007;92(2):F8388.

5. Maisels MJ. Managing the jaundiced newborn: a persistent challenge. CMAJ. 2015;187(5):335-343.

6. Stokowski LA. Fundamentals of phototherapy for neonatal jaundice. Adv Neonatal Care. 2011;11(5 Suppl):S10-21.

7. Gottstein R, Cooke RW. Systematic review of intravenous immunoglobulin in haemolytic disease of the newborn. Arch Dis Child Fetal Neonatal Ed. 2003;88(1):F6-10.

8. Walsh SA, Yao N, El-Khuffash A, Twomey A, Molloy EJ. Efficacy of intravenous immunoglobulin in the management of haemolytic disease of the newborn. Ir Med J. 2008;101(2):46-48.

9. Rubo J, Albrecht K, Lasch P, Laufkotter E, Leititis J, Marsan D, Niemeyer B, et al. High-dose intravenous immune globulin therapy for hyperbilirubinemia caused by Rh hemolytic disease. J Pediatr. 1992;121(1):93-97.

10. Alpay F, Sarici SU, Okutan V, Erdem G, Ozcan O, Gokcay E. High-dose intravenous immunoglobulin therapy in neonatal immune haemolytic jaundice. Acta Paediatr. 1999;88(2):216-219.

11. American Academy of Pediatrics Subcommittee on Hyperbilirubinemia. Management of hyperbilirubinemia in the newborn infant 35 or more weeks of gestation. Pediatrics. 2004;114(1):297-316.

12. Keir AK, Dunn M, Callum J. Should intravenous immunoglobulin be used in infants with isoimmune haemolytic disease due to ABO incompatibility? J Paediatr Child Health. 2013;49(12):1072-1078.

13. Kara S, Ulu-ozkan H, Yilmaz Y, Arikan FI, Dilmen U, Bilge YD. Necrotizing enterocolitis in a newborn following intravenous immunoglobulin treatment for haemolytic disease. J Coll Physicians Surg Pak. 2013;23(8):598600 .

14. Beken S, Hirfanoglu I, Turkyilmaz C, Altuntas N, Unal $\mathrm{S}$, Turan $\mathrm{O}$, Onal $\mathrm{E}$, et al. Intravenous immunoglobulin $\mathrm{G}$ treatment in $\mathrm{ABO}$ hemolytic disease of the newborn, is it myth or real? Indian J Hematol Blood Transfus. 2014;30(1):12-15.

15. Cynthia A Mundy, Jatinder Bhatia. Immunoglobulin transfusion in hemolytic disease of the newborn: place in therapy. International Journal of Clinical Transfusion Medicine. 2015;3:41-45.

16. Ohlsson A, Lacy JB. Intravenous immunoglobulin for preventing infection in preterm and/or low birth weight infants. Cochrane Database Syst Rev. 2013;7:CD000361.

17. Jelkmann W. Proinflammatory cytokines lowering erythropoietin production. J Interferon Cytokine Res. 1998;18(8):555-559.

18. Saleh Al-Alaiyan, Husain Alsaid Ahmad, Fahad Al-Hazzani, et al. Effects of intravenous human immunoglobulin on late hyporegenerative anemia secondary to rhesus hemolytic disease of the newborn. IJPAM. 2014;11:73-77. 